\title{
Fisher's Fundamental Theorem of Natural Selection Revisited
}

\author{
Sabin Lessard ${ }^{1}$ \\ Département de mathématiques et de statistique, \\ Université de Montréal, C.P. 6128, Succursale Centre-Ville, \\ Montréal, Québec, Canada H3C $3 J 7$
}

Received June 19, 1996

\begin{abstract}
W. J. Ewens, following G. R. Price, has stressed that Fisher's fundamental theorem of natural selection about the increase in mean fitness is of general validity without any restrictive assumptions on the mating system, the fitness parameters, or the numbers of loci and alleles involved, but that it concerns only a partial change in mean fitness. This partial change is obtained by replacing the actual genotypic fitnesses by the corresponding additive genetic values and by keeping these values fixed in the change of the mean with respect to changes in genotype frequencies. We propose an alternate interpretation for this partial change which uses partial changes in genotype frequencies directly consequent on changes in gene frequencies, the fitness parameters being kept constant. We argue that this interpretation agrees more closely with Fisher's own explanations. Moreover, this approach leads to a decomposition for the total change in mean fitness which explains, unifies, and extends previous decompositions. We consider a wide range of models, from discrete-time selection models with nonoverlapping generations to continuous-time models with overlapping generations and age effects on viability and fecundity, which is the original framework for Fisher's fundamental theorem. (C) 1997 Academic Press
\end{abstract}

\section{INTRODUCTION}

Fisher (1930, p. 35; 1958, p. 37) stated his Fundamental Theorem of Natural Selection (FTNS) for a continuoustime model in the form:

\footnotetext{
"The rate of increase in fitness of any organism at any time is equal to its genetic variance in fitness at that time."
}

There were no restrictive assumptions on the mating system, the fitness parameters, or the genetic background. It was shown later by Kimura (1958) that, for such a model, the total rate of change in population fitness involves two additional terms, the first one due to

\footnotetext{
${ }^{1}$ Supported in part by a NSERC grant from the Government of Canada and a FCAR grant from the Government of Quebec. E-mail: lessards@ere.umontreal.ca.
}

changes in individual fitness parameters, if any, and the second one due to dominance and epistatic effects of genes on fitness which come into play unless there is random combination of genes in individuals. Moreover, unlike the genetic variance in fitness, either of these two terms may be negative and therefore may cause a decrease in population fitness. This could suggest that the FTNS is only an approximate result for the total change in population fitness. Another view is to consider that the FTNS is rigorously exact if it is correctly interpreted as claimed by Fisher $(1958$, p. 38$)$ : "The rigour of the demonstration requires that the terms employed should be used strictly as defined." We adhere entirely to this view.

The terms employed by Fisher (1958) in his derivation of the FTNS are average excess, average effect, genetic variance, Malthusian parameter, and reproductive value. 
It is clear that what is meant by genetic variance, that is, the variance of the genetic value which is "the value of the genotype as best predicted from the genes present" (idem, p. 35), is what we call today genic variance or additive genetic variance. Given any quantitative individual measurement, this is the variance of the additive genetic value or genic value, which is the sum of the average effects of the genes present in the individual. With multiple allelomorphism, the average effect, also called today the additive effect, is defined by Fisher as "the amount of the difference produced, on the average, in the total measurement of the population, [by] substituting any chosen gene for a random selection of the genes homologous with it" (idem, p. 35). The values of the average effects are obtained by the least-squares method as "the best additive system for predicting the genotypic value from the actual genes present in any individual" (idem, p. 35). The avarege effect must be distinguished from the average excess which is the difference between the average measurement in "all the individuals carrying any one gene, counting the homozygotes twice" (idem, p. 34) and the average measurement in the whole population.

Average effect and average excess can be applied to the Malthusian parameter: "Any group of individuals selected as bearers of a particular gene, and consequently the genes themselves, will have [relative] rates of increase which may differ from the average" (idem, p. 37). The relative rate of increase, which represents a decrease if it is negative, corresponds to a logarithmic rate of increase in a population with overlapping generations and is defined by the Malthusian parameter "implicit in any given system of rates of death and reproduction" (idem, p. 26). This parameter satisfies Lotka's (1922) equation for the given system and "measures the relative rate of increase or decrease of a population when in the steady state [with respect to age distribution] appropriate to any such system" (idem, p. 26). This rate has to be distinguished from the expectation of offspring of a newlyborn individual, but "must be positive if the expectation of offspring exceeds unity, and must be negative if it falls short of unity" (idem, p. 26). Moreover, "owing to bisexual reproduction $[\ldots]$, each living offspring will be credited to both parents, and it will seem proper to credit each with one half in respect of each offspring" (idem, p. 24).

There is an alternate interpretation for the Malthusian parameter: if individuals of each age are assigned an appropriate value, called its reproductive value and defined as "the present value of [its] future offspring" (idem, p. 27), then "whatever may be the age constitution of a population, its total reproductive value will increase or decrease according to the correct Malthusian rate" (idem, p. 29). Therefore the Malthusian parameter "measures fitness by the objective fact of representation in future generations" (idem, p. 37). Moreover, it "will in general be different for each different genotype" (idem, p. 50), to which correspond specific rates of death and reproduction.

Measuring fitness by the Malthusian parameter, the FTNS concerns "the rate of increase in fitness due to all changes in gene ratio" (idem, p. 37) or "the rate of increase in the mean value $[\ldots]$ produced by Natural Selection" (idem, pp. 45-46). This rate of increase in population fitness, or the mean fitness, "is exactly equal to the genetic variance of fitness $[\ldots]$ which the population exhibits" (idem, p. 37). Changes in gene ratio and effects of natural selection refer only to changes in the gene frequency structure. In order to ease the interpretation of the FTNS, the gene frequencies "should strictly be evaluated at any instant by the enumeration, not necessarily of the census population, but of all individuals having reproductive value, weighted according to the reproductive value of each. [Moreover] the theorem is exact only for idealized populations, in which fortuitous fluctuations in genetic composition have been excluded" (idem, p. 38). Therefore the population is assumed to be infinite and, in calculating averages, the individuals are weighted by their reproductive value according to their age.

Several passages in Fisher's (1958) book suggest that the FTNS accounts only for a partial change in population fitness. The first one concerns the effects of genetic dominance in relation with the mating pattern: "In addition to the genetic variance $[\ldots]$, a second element comprised in the total genotypic variance $[\ldots]$, ascribable to dominance, is also in a sense capable of exerting evolutionary effects, not through any direct effect on the gene ratios, but through its possible influence on the breeding system" (idem, p. 40). The second passage is about deterioration in the environment: "Against the action of Natural Selection in constantly increasing the fitness of every organism [...], is to be set off the very considerable item of the deterioration of its inorganic and organic environment" (idem, p. 45). Such deterioration is typically caused by mutations, geological and climatological changes, and more numerous enemies and competitors. The last passage concerns changes in population size: "An increase in numbers of any organism will impair its environment" (idem, p. 45). This is the deleterious effect of overcrowding which will be responsible for the population fitness not to always increase or decrease but rather to approach a constant value. 
As explained by Kimura (1958, p. 165), deterioration in environment in a broad sense might include not only any change in the fitnesses of the different possible genotypes under changing external (physical and biological) conditions, but also any change in the mating system in conjunction with dominance and epistatic effects of genes defining the genetical environment. Price (1972, p. 133) incorporates the change in the mean fitness due to population pressure into the change due to what he calls the environment change effects. Moreover, he attributes the change due to natural selection to "the additive or linear effects of changes in gene frequencies" (idem, p. 130). He interprets this change as the change in the mean (additive) genetic value, keeping fixed the average effects, or more precisely, the partial regression coefficients of fitness on the frequencies of the allelomorphic genes in the members of the population, which are linearly related to the average effects. But he doubts that Fisher would have accepted this interpretation since, although Fisher could have presented his theorem in this way, he did not. Moreover, in Price's clarification of Fisher's derivation of the FTNS, the central statement that "any increase $d p$ in the frequency of the chosen gene will be accompanied by an increase $2 \alpha d p$ in the average fitness of the species" (Fisher, 1958, p. 37), where $\alpha$ is the average effect of the chosen gene, is simply replaced by an equation (Price, 1972, Eq. 5.8) which states that $2 \alpha$ is the partial derivative of the mean fitness with respect to the frequency of the chosen gene, keeping the environment unchanged. This equation is fundamental to showing that the rate of change in the mean fitness due to natural selection is given by the additive genetic variance in fitness. Finally, Price (1972, p. 139) does not use weighting by reproductive value the way Fisher suggests since he thinks that this leads to a "mathematical inconsistency" (Price and Smith, 1972).

Ewens (1989) accepts Price's (1972) interpretation of the FTNS and claims that the FTNS is not specifically a continuous-time result. He introduces the partial change in mean fitness obtained by replacing the fitnesses of the different possible genotypes in the mean by the corresponding additive genetic values and by keeping these values fixed in the change of the mean with respect to changes in genotype frequencies. Then he shows that, with discrete, nonoverlapping generations, the partial change in mean fitness from the current generation to the next generation is equal to the additive genetic variance in fitness divided by the mean fitness. This is shown to be true with viability selection from conception to maturity determined at one or several loci (Ewens, 1989; see Ewens, 1992, and Castilloux and Lessard, 1995, for correction of Eq. 26) as long as mating and reproduction do not change gene frequencies from the current generation at the time of maturity to the next generation at the time of conception. This is also true with fecundity selection determined at one or several loci if mating does not change gene frequencies in the current generation from the time of conception to the time of reproduction and if neither meiotic drive nor gametic selection takes place (Lessard and Castilloux, 1995).

Fisher (1941) has explained the meaning of his FTNS in a paper entitled "Average Excess and Average Effect of a Gene Substitution." This title should suggest that the key elements to understanding the fundamental theorem are to be found in these two concepts. Fisher (1941, pp. 53-54) writes: "The average excess of any measurement in respect of any gene substitution $[\ldots]$ is defined directly in terms of the numbers and mean measurements of the $[\ldots]$ genotypes $[\ldots]$ into which the population may be divided. [It] is not, however, to be ascribed to the gene substitution. [The] average effect of a given gene substitution [is conceived] as the actual increase in the total of the measurements of a population, when without change in the environment, or in the mating system, the gene substitution is experimentally brought about, as it might be by mutation. [...] A change in the proportion of any pair of genes itself constitutes a change in the environment in which individuals of the species find themselves. [The] effect that is wanted is only that due to the change in the frequencies of the different possible genotypes, not including any change in the average measurement of a given genotype, which the change in gene frequency may in fact bring about. [Since] the number of genotypes greatly exceeds the number of gene ratios on which their frequencies depend, we are concerned only with those changes of genotype frequency directly consequent on the proposed change of gene ratio, in the actual condition of the population. [...] The direct mathematical measure of the average effect of a proposed gene substitution is the partial regression, in the population as actually constituted, of the genotypic measurement on the numbers 0,1 , or 2 of the allelomorphic genes in each genotype."

The average effect of a gene substitution on a quantitative character must be such that, in a constant environment, including a "constant genic environment" (idem, p. 56), the increase in the total measurement of the population will be just the average effect times the number of substituted genes. Constant environment guarantees fixed genotypic values and constant genic environment is assured when "the change in genotypic frequencies [is] ascribable only to change in gene ratio" (idem, p. 56). With two allelomorphic genes determining 
a single factor at an autosomal locus in a diploid population, this is shown to be the case when

$$
\frac{d P}{P}+\frac{d R}{R}=\frac{2 d Q}{Q},
$$

where $2 Q$ is the frequency of the heterozygote ( $Q$ being the frequency of each of the ordered heterozygotes), $P$ and $R$ are the frequencies of the two homozygotes, and $2 d Q, d P$, and $d R$ represent instantaneous changes in these frequencies. This holds when the ratio

$$
\frac{Q^{2}}{P R}=\lambda
$$

remains constant. When the different genotypes are in Hardy-Weinberg proportions, this ratio $\lambda$ is identically equal to 1 .

With the above definition of average effect applied to fitness in a diploid population, "the increase of average fitness of the population ascribable to a change in gene frequency $d p$ will be $2 \alpha d p$ " (idem, p. 57), where $\alpha$ is the average effect of the substituted gene. On the other hand, if we "choose as the metrical variable characteristic of the different genotypes, the Malthusian parameter of population increase $[\ldots]$ measuring the survival value of each" (idem, p. 56), which represents fitness, then the average excess of any substituted gene is related to its change in frequency due to natural selection such that "the rate of increase in the average value of the Malthusian parameter ascribable to natural selection $[\ldots]$ will be [...] equal to the genetic variance of fitness" (idem, p. 57), if all allelomorphic genes of each factor and all factors are taken into consideration.

Kempthorne (1957, Chap. 16), recognizing that he has been unable to follow Fisher's derivation of the FTNS, analyzes a one-locus model assuming discrete, nonoverlapping generations and constant reproductive values defined as the numbers of offspring minus 1 , crediting each parent with one-half of its actual offspring. Identifying fitness with the natural logarithm of 1 plus the reproductive value, he ascertains Fisher's theorem for the case of two alleles "provided that the mating system is such that $Q^{2} / P R$ remains constant" (Kempthorne, 1957 , p. 360). He proves this assertion by showing that the change in the mean dominance deviation in reproductive value through the changes in the genotypic frequencies, keeping the dominance deviations fixed, is 0 when

$$
\frac{2 \Delta Q}{Q}=\frac{\Delta P}{P}+\frac{\Delta R}{R}
$$

which is a discrete-time analogue of Eq. (1), where $2 \Delta Q$, $\Delta P$, and $\Delta R$ represent changes from the current generation to the next generation in the genotypic frequencies $2 Q, P$, and $R$, and by assuming weak selection, which guarantees small changes in the genotypic frequencies and simple approximate relations between fitness and reproductive value as defined above.

Kempthorne (1957) also gives, under the same assumptions, an approximate expression for the total change in mean fitness for the case of multiple alleles at a single locus, using multiallelic analogues of Fisher's (1941) ratio $\lambda$ to keep track of changes in the mating system. Kimura (1958) obtains an equivalent expression for a continuous-time model, using coefficients of departure from random combinations of genes, represented by $\theta$ and defined as the frequencies of the ordered genotypes divided by the products of the corresponding gene frequencies. The formula is extended to take into account changes in fitness parameters and genetic determination at two or more loci. For one-locus models, the formula is exact in continuous time, while it is only approximate in discrete time, since smaller-order terms are ignored in this case. Li (1969) gives an exact formula for this case, but he assumes random mating. Crow (1979) incorporates weighting by reproductive values according to age for the case of age-structured populations. For twolocus and multilocus models, the formula can be extended to an exact formula, not only for the change in mean fitness but also for the change in the mean of any quantitative character correlated with fitness, by adding extra terms to Kimura's formula in continuous time to take into account all epistatic effects (see, e.g., Crow and Nagylaki, 1976, and Nagylaki, 1989, for the case of two loci), or by assuming no epistasis and random mating in discrete time (Nagylaki, 1989, 1991).

A "simplied version of Fisher's fundamental theorem of natural selection" for populations undergoing discrete, nonoverlapping generations, which is due to $\mathrm{Li}$ (1955), states that the increase in the mean genotypic fitness from the beginning to the end of the current generation as a result of differential survival rates is equal to the genotypic variance in fitness, or the total variance in fitness, at the beginning of the current generation divided by the mean fitness (that is, the total coefficient of variation in fitness). This version "excludes the round of mating, which is included in Fisher's theorem" as claimed by Turner (1970) in his review on changes in mean fitness under natural selection. Nevertheless, this is interpreted differently by Denniston (1978), who includes the round of mating but defines the fitness of a genotype as the ratio, in numbers, of individuals of this genotype in the next generation to those in the current generation. In a 
continuous-time version, this corresponds to defining the Malthusian parameter for a genotype as its geometric rate of increase or decrease in number. This definition has been occasionally attributed to Fisher by some authors (see, e.g., Kimura, 1956, and Charlesworth, 1970) and even used from time to time (see, e.g., Emlen, 1970), but it remains unconventional. Denniston (1978) studies the implications of using this definition on the decomposition of the mean fitness and the decomposition of the mean of any quantitative character correlated with fitness.

For a recent review on the FTNS with historic perspectives, we refer the reader to Edwards (1994). See also Frank and Slatkin (1992) for the evolutionary significance of the FTNS in an ecological context.

In this paper, we study the change in mean fitness, formalizing ideas that have been put forward by Fisher (1941), but that are still misunderstood. The starting point is the analysis of the average excess and average effect of a gene substitution with respect to genotypic fitness, defined as the mean number of offspring produced by individuals of each genotype from the beginning to the end of the current generation, and genotypic growth rate, defined as the ratio, in numbers, of individuals of each genotype in the next generation to those of the same genotype in the current generation, in populations undergoing discrete, nonoverlapping generations. Then we propose an interpretation of the FTNS which is more consistent than ever with Fisher's writings and we deduce a decomposition for the total change in mean fitness which extends, unifies, and enlightens previous decompositions. We begin with a basic discrete-time, multiallele multilocus selection model with nonoverlapping generations, and we end up with a continuous-time model with overlapping generations and age effects on viability and fecundity, which corresponds to the original framework for Fisher's (1930) FTNS.

\section{BASIC MODEL AND DEFINITIONS}

Let zygotic selection in an infinite diploid sexual population be determined at $L$ autosomal loci with possible alleles $A_{1}, \ldots, A_{n_{1}}$ at locus $1, A_{n_{1}+1}, \ldots, A_{n_{2}}$ at locus $2, \ldots$, and $A_{n_{L-1}+1}, \ldots, A_{n_{L}}$ at locus $L$. Generations of the population are discrete and nonoverlapping, segregation of genes is Mendelian and there is no gametic selection. The recombination scheme is general and mating is not assumed to be random.

A typical gamete is represented by a $n_{L}$-dimensional vector

$$
\xi=\left(\xi_{1}, \ldots, \xi_{n_{1}}, \xi_{n_{1}+1}, \ldots, \xi_{n_{2}}, \ldots, \xi_{n_{L-1}+1}, \ldots, \xi_{n_{L}}\right),
$$

where $\xi_{i}=1$ if allele $A_{i}$ is present at locus $l$, for $n_{l-1}$ $+1 \leqslant i \leqslant n_{l}$ and $1 \leqslant l \leqslant L$ (with $n_{0}=0$ ), and $\xi_{i}=0$ otherwise. Since a genotype corresponds to a couple of parental gametes, a typical genotype is represented by a vector

$$
g=\left(\xi^{(m)}, \xi^{(f)}\right),
$$

where $\xi^{(m)}$ is a paternal gamete and $\xi^{(f)}$ is a maternal gamete. Note that $g$ is an ordered genotype since we keep track of the origins of the gametes. The frequency of $g$ in the population at the beginning of the current generation is denoted by $x^{(g)}$. It is assumed that

$$
x^{(g)}>0,
$$

for every possible $g$.

Let the fitness of $g$, denoted by $w^{(g)}$, be interpreted as the mean number of offspring produced by individuals whose genotype is $g$, from the beginning to the end of the current generation. In this interpretation, we use the convention that every successful gamete counts for half an offspring since every offspring is made of two parental gametes. Moreover, this definition incorporates viability as well as fecundity differences.

Fitness as defined above is a Darwinian fitness since it measures "success in leaving progeny" (Darwin, 1956, p. 64). Moreover, the mean fitness in the population at the current generation is

$$
\bar{w}=\sum_{g} x^{(g)} w^{(g)}
$$

Note that $\bar{w}$ gives the growth rate of the population from the current generation to the next generation since $\bar{w}$ is the mean number of offspring per individual of the current generation. Note also that

$$
x^{(g)^{*}}=\frac{x^{(g)} w^{(g)}}{\bar{w}}
$$

is the frequency of $g$ in the parents of the offspring at the beginning of the next generation, counted as many times as their mean number of offspring. This is the probability that a gene chosen at random at any of the given loci in an offspring chosen at random at the beginning of the next generation comes from a parent of genotype $g$.

The frequency of $g$ in the offspring at the beginning of the next generation, denoted by $x^{(g)^{\prime}}$, is generally different from $x^{(g)^{*}}$ because of sexual reproduction with 
segregation and recombination of genes. Nevertheless, it can always be written in a form analogous to (5), that is,

$$
x^{(g)^{\prime}}=\frac{x^{(g)} W^{(g)}}{\bar{W}},
$$

where $W^{(g)}$ represents the growth rate in the number of individuals of genotype $g$ from the current generation to the next generation, and

$$
\bar{W}=\sum_{g} x^{(g)} W^{(g)}=\bar{w} .
$$

The genotypic growth rate $W^{(g)}$ is mathematically defined by the formula

$$
W^{(g)}=\left[\frac{x^{(g)^{\prime}}}{x^{(g)}}\right] \bar{w}
$$

which corresponds to the ratio, in numbers, of individuals of genotype $g$ in the next generation to those in the current generation. This ratio may be a complex function of the state of the population involving many parameters for survival, mating, and fecundity, not to mention recombination. But it can be used as a definition, though unconventional, of fitness (see Denniston, 1978, and references therein). Equation (7) means that the mean genotypic growth rate is equal to the growth rate of the population.

\section{CHANGES IN GENE FREQUENCIES}

If the frequency of an allele $A_{i}$ at a given locus in genotype $g$ is represented by $f_{i}^{(g)}$ (with possible values 0 , $1 / 2$, and 1 ), then the frequency of $A_{i}$ in the population at the beginning of the current generation, denoted by $p_{i}$, is

$$
p_{i}=\bar{f}_{i}=\sum_{g} x^{(g)} f_{i}^{(g)} .
$$

Note that

$$
\sum_{i=n_{l-1}+1}^{n_{l}} f_{i}^{(g)}=1 \quad \text { and } \quad \sum_{i=n_{l-1}+1}^{n_{l}} p_{i}=1 \text {, }
$$

for $l=1, \ldots, L$.

The frequency of $A_{i}$ in the offspring at the beginning of the next generation, denoted by $p_{i}^{\prime}$, will be the same as the frequency of $A_{i}$ in the parents of those offspring, counted as many times as their mean number of offspring, since we assume Mendelian segregation of genes and no gametic selection. Then we have

$$
p_{i}^{\prime}=\sum_{g} x^{(g)^{*}} f_{i}^{(g)}
$$

Using (5) and (8), we find that the change in the frequency of $A_{i}$ from the current generation to the next generation is

$$
\Delta p_{i}=p_{i}^{\prime}-p_{i}=\frac{\operatorname{cov}\left(f_{i}, w\right)}{\bar{w}},
$$

where

$$
\operatorname{cov}\left(f_{i}, w\right)=\sum_{g} x^{(g)} f_{i}^{(g)} w^{(g)}-\bar{f}_{i} \bar{w} .
$$

This is Price's (1970) covariance formula for the change in the frequency of an allele, which is a special case, in discrete time, of the Secondary Theorem of Natural Selection (STNS) proposed by Robertson (1966) for the change in a quantitative character correlated with fitness. The above formula for the change in the frequency of an allele was also noted by $\mathrm{Li}$ (1967), but under the assumption of random union of gametes. We will refer to it as the Li-Price covariance formula.

The frequency of $A_{i}$ in the offspring at the beginning of the next generation is also given by the equation

$$
p_{i}^{\prime}=\sum_{g} x^{(g)^{\prime}} f_{i}^{(g)}
$$

Then, using (6) and (8), we get also

$$
\Delta p_{i}=\frac{\operatorname{cov}\left(f_{i}, W\right)}{\bar{W}} .
$$

Therefore the Li-Price covariance formula for the change in the frequency of an allele holds if fitness is defined by (6). Note that, in this case, the assumptions of Mendelian segregation and no gametic selection do not have to be made.

Note also that Eqs. (10) and (11) can be written in the form

$$
\bar{w} \Delta p_{i}=p_{i} a_{i},
$$

where $a_{i}$ stands for the average genotypic excess in fitness of allele $A_{i}$ as defined by Fisher (1958, p. 34). This excess 
is the same if fitness is defined by (5) or (6), as already noted by Denniston (1978), and is given by

$$
a_{i}=\frac{\operatorname{cov}\left(f_{i}, w\right)}{\bar{f}_{i}}=\frac{\operatorname{cov}\left(f_{i}, W\right)}{\bar{f}_{i}} .
$$

This is the deviation of the marginal fitness of $A_{i}$ from the mean fitness in the population. Equation (12) is a discrete-time analogue of an equation in continuous time given by Fisher (1958, p. 37). Therefore the Li-Price covariance formula (10) can be traced back to Fisher's works and, for this reason, it will be called the Fisher-LiPrice covariance formula.

\section{ADDITIVE ALLELIC EFFECTS ON FITNESS}

Following Fisher (1958, p. 35), the average effects, or additive effects, of the genes on fitness and the corresponding genetic values, or additive fitnesses, of the genotypes are obtained by the least-squares method. We write the fitness $w^{(g)}$, defined by (5), minus its mean in the form

$$
w^{(g)}-\bar{w}=\alpha^{(g)}+\delta^{(g)},
$$

where

$$
\alpha^{(g)}=2 \sum_{i} f_{i}^{(g)} \alpha_{i}
$$

is such that

$$
\sigma_{\delta}^{2}=\sum_{g} x^{(g)}\left[\delta^{(g)}\right]^{2}
$$

is minimum under the constraints

$$
\sum_{i=n_{l-1}+1}^{n_{l}} p_{i} \alpha_{i}=0, \quad \text { for } \quad l=1, \ldots, L .
$$

Then $\alpha_{i}$ is the additive effect of $A_{i}$ on $w, \alpha^{(g)}$ is the corresponding additive fitness of $g$, and $\delta^{(g)}$ is a residual addend incorporating dominance and epistatic effects of genes. Note that the constraints (16) imply that

$$
\bar{\alpha}=\sum_{g} x^{(g)} \alpha^{(g)}=0
$$

and then, by (13),

$$
\bar{\delta}=\sum_{g} x^{(g)} \delta^{(g)}=0 .
$$

Defining the function

$$
S=\sigma_{\delta}^{2}+4 \sum_{l=1}^{L} \lambda_{l} \sum_{i=n_{l-1}+1}^{n_{l}} p_{i} \alpha_{i},
$$

where $\lambda_{1}, \ldots, \lambda_{L}$ are Lagrange multipliers, the partial derivative of $S$ with respect to $\alpha_{i}$ is 0 if and only if

$$
\sum_{g} x^{(g)} f_{i}^{(g)} \delta^{(g)}-p_{i} \lambda_{l}=0
$$

where $n_{l-1} \leqslant i \leqslant n_{l}$ and $1 \leqslant l \leqslant L$. Summing over $i$ from $n_{l-1}+1$ to $n_{l}$ in (19) and using (9) yield

$$
\lambda_{l}=\bar{\delta}
$$

for $l=1, \ldots, L$. Then (19) becomes

$$
\operatorname{cov}\left(f_{i}, \delta\right)=0 \text {. }
$$

Owing to (13) and (14), this is equivalent to

$$
\operatorname{cov}\left(f_{i}, w\right)=\operatorname{cov}\left(f_{i}, \alpha\right)=2 \sum_{j} \operatorname{cov}\left(f_{i}, f_{j}\right) \alpha_{j},
$$

where

$$
\operatorname{cov}\left(f_{i}, f_{j}\right)=\sum_{g} x^{(g)} f_{i}^{(g)} f_{j}^{(g)}-p_{i} p_{j}
$$

Using (16), Eq. (21) can also be written in the form

$$
\operatorname{cov}\left(f_{i}, w\right)=2 \sum_{j} \bar{f}_{i} f_{j} \alpha_{j}
$$

where

$$
\overline{f_{i} f_{j}}=\sum_{g} x^{(g)} f_{i}^{(g)} f_{j}^{(g)}
$$

This is the probability that two genes chosen at random and independently, in the same individual chosen at random in the population at the beginning of the current generation, the first one at the locus of $A_{i}$ and the second one at the locus of $A_{j}$, will be $A_{i}$ and $A_{j}$ in this order.

From (14) and (20), we have

$$
\operatorname{cov}(\alpha, \delta)=2 \sum_{i} \operatorname{cov}\left(f_{i}, \delta\right) \alpha_{i}=0,
$$


from which

$$
\sigma_{w}^{2}=\sigma_{\alpha}^{2}+\sigma_{\delta}^{2}
$$

where

$$
\sigma_{w}^{2}=\sum_{g} x^{(g)}\left[w^{(g)}-\bar{w}\right]^{2}
$$

is the total variance of $w$,

$$
\sigma_{\alpha}^{2}=4 \sum_{i, j} \operatorname{cov}\left(f_{i}, f_{j}\right) \alpha_{i} \alpha_{j}=4 \sum_{i, j} \overline{f_{i} f_{j}} \alpha_{i} \alpha_{j}
$$

is the corresponding additive genetic variance, and $\sigma_{\delta}^{2}$ is the residual variance given in (15).

Finally, combining Eqs. (21) and (22) with the FisherLi-Price covariance formula (10) leads to

$$
\bar{w} \Delta p_{i}=2 \sum_{j} C_{i j} \alpha_{j}
$$

where

$$
C_{i j}=\operatorname{cov}\left(f_{i}, f_{j}\right) \quad \text { for all } i, j
$$

or

$$
C_{i j}=\bar{f}_{i} f_{j} \quad \text { for all } i, j .
$$

In vector notation, $(25)$ for $i=1, \ldots, n_{L}$ takes the form

$$
\bar{w} \Delta \mathbf{p}=2 \mathbf{C \alpha},
$$

from which the additive genetic variance (24) is given by

$$
\sigma_{\alpha}^{2}=4 \boldsymbol{\alpha}^{T} \mathbf{C} \boldsymbol{\alpha}=2 \bar{w} \boldsymbol{\alpha}^{T} \Delta \mathbf{p} .
$$

This equation, with the constraints (16), characterizes the additive allelic effects on $w$. It can be shown that $\boldsymbol{\alpha}$ satisfying (26) and (16) exists and is unique. Moreover, the critical point $\boldsymbol{\alpha}$ is actually a minimum point of $S$ under the constraints (16) (see Lessard and Castilloux, 1995).

Owing to (7) and (11), we get, surprisingly, the same equation (26) if the genotypic growth rate $W^{(g)}$ defined by (6) is used as fitness. We conclude that the additive allelic effects on $W$ are the same as the additive allelic effects on $w$. Therefore, if we write

$$
W^{(g)}-\bar{W}=\alpha^{(g)}+\varepsilon^{(g)},
$$

where $\alpha^{(g)}$ is the additive fitness defined by (14), (16), and (26), then the residual addend $\varepsilon^{(g)}$ has mean $\bar{\varepsilon}=0$ and its variance

$$
\sigma_{\varepsilon}^{2}=\sum_{g} x^{(g)}\left[\varepsilon^{(g)}\right]^{2}
$$

is minimum under the constraints (16). This entails

$$
\operatorname{cov}\left(f_{i}, \varepsilon\right)=0
$$

for every $i$, and then

$$
\operatorname{cov}(\alpha, \varepsilon)=0
$$

from which the total variance of $W$ satisfies

$$
\sigma_{W}^{2}=\sigma_{\alpha}^{2}+\sigma_{\varepsilon}^{2}
$$

\section{CHANGE IN MEAN FITNESS}

The total change in the mean fitness of the population from the current generation to the next generation can be decomposed as

$$
\begin{aligned}
\Delta \bar{w}= & \sum_{g}\left[\Delta x^{(g)}\right] w^{(g)}+\sum_{g} x^{(g)}\left[\Delta w^{(g)}\right] \\
& +\sum_{g}\left[\Delta x^{(g)}\right]\left[\Delta w^{(g)}\right],
\end{aligned}
$$

where $\Delta x^{(g)}$ and $\Delta w^{(g)}$ stand for the total changes in the frequency and the fitness of genotype $g$, respectively, from the current generation to the next generation. The last two terms correspond to partial changes due at least in part to changes in the environment, since they vanish if the genotypic fitnesses are constant from the current generation to the next generation, while the first term represents a partial change due only to changes in genotype frequencies.

From Eq. (6), the change in the frequency of genotype $g$ from the current generation to the next generation can be expressed as

$$
\Delta x^{(g)}=x^{(g)^{\prime}}-x^{(g)}=\frac{x^{(g)}\left[W^{(g)}-\bar{W}\right]}{\bar{W}}
$$

and, owing to Eq. (28) on the growth rate of genotype $g$, can be decomposed as

$$
\Delta x^{(g)}=\left[\Delta x^{(g)}\right]_{\alpha}+\left[\Delta x^{(g)}\right]_{\varepsilon},
$$


where

$$
\left[\Delta x^{(g)}\right]_{\alpha}=\frac{x^{(g)} \alpha^{(g)}}{\bar{W}}
$$

and

$$
\left[\Delta x^{(g)}\right]_{\varepsilon}=\frac{x^{(g)} \varepsilon^{(g)}}{\bar{W}}
$$

$\bar{W}$ being the growth rate of the population from the current generation to the next generation, $\alpha^{(g)}$ being the additive growth rate of genotype $g$, and $\varepsilon^{(g)}$ being the associated residual addend. The first term in this decomposition represents the change ascribable only to changes in gene frequencies. Note that when $\varepsilon^{(g)}=0$, the relative change in the frequency of genotype $g$ from the current generation to the next generation, namely $\Delta x^{(g)} / x^{(g)}$, is given by $\alpha^{(g)} / \bar{W}$, which is linear with respect to gene frequencies in $g$ owing to (14).

The above decomposition for the change in genotype frequencies leads to the following one for the first term on the right side of (32),

$$
\sum_{g}\left[\Delta x^{(g)}\right] w^{(g)}=\sum_{g}\left[\Delta x^{(g)}\right]_{\alpha} w^{(g)}+\sum_{g}\left[\Delta x^{(g)}\right]_{\varepsilon} w^{(g)},
$$

where, owing to (7), (17), (18), (23), and (30), we have

$$
\begin{aligned}
\sum_{g}\left[\Delta x^{(g)}\right]_{\alpha} w^{(g)} & =\sum_{g} \frac{x^{(g)} \alpha^{(g)} w^{(g)}}{\bar{W}} \\
& =\sum_{g} \frac{x^{(g)} \alpha^{(g)}\left[\bar{w}+\alpha^{(g)}+\delta^{(g)}\right]}{\bar{w}} \\
& =\frac{\bar{\alpha} \bar{w}+\sigma_{\alpha}^{2}+\operatorname{cov}(\alpha, \delta)}{\bar{w}}=\frac{\sigma_{\alpha}^{2}}{\bar{w}}
\end{aligned}
$$

and

$$
\begin{aligned}
\sum_{g}\left[\Delta x^{(g)}\right]_{\varepsilon} w^{(g)} & =\sum_{g} \frac{x^{(g)} \varepsilon^{(g)} w^{(g)}}{\bar{W}} \\
& =\sum_{g} \frac{x^{(g)} \varepsilon^{(g)}\left[\bar{w}+\alpha^{(g)}+\delta^{(g)}\right]}{\bar{w}} \\
& =\frac{\bar{\varepsilon} \bar{w}+\operatorname{cov}(\varepsilon, \alpha)+\operatorname{cov}(\varepsilon, \delta)}{\bar{w}} \\
& =\frac{\operatorname{cov}(\varepsilon, \delta)}{\bar{w}} .
\end{aligned}
$$

The first term is the change in the mean fitness due only to changes in gene frequencies. The second term is a change due to changes in the genic environment.

Similarly, we get, for the last term on the right side of (32),

$$
\sum_{g}\left[\Delta x^{(g)}\right]\left[\Delta w^{(g)}\right]=\frac{\operatorname{cov}(\alpha, \Delta w)}{\bar{w}}+\frac{\operatorname{cov}(\varepsilon, \Delta w)}{\bar{w}},
$$

so that the total change in mean fitness takes the form

$$
\Delta \bar{w}=\frac{\sigma_{\alpha}^{2}}{\bar{w}}+\frac{\operatorname{cov}(\varepsilon, \delta)}{\bar{w}}+\overline{\Delta w}+\frac{\operatorname{cov}(\alpha, \Delta w)}{\bar{w}}+\frac{\operatorname{cov}(\varepsilon, \Delta w)}{\bar{w}},
$$

where

$$
\overline{\Delta w}=\sum_{g} x^{(g)}\left[\Delta w^{(g)}\right]
$$

is the mean of the changes in the genotypic fitnesses from the current generation to the next generation. This is an exact decomposition for discrete-time models with any number of loci involved, which extends, using different variables, Kempthorne's (1957) approximate decomposition for one-locus models (see Section 8.2 for details). The first term in this decomposition is interpreted in the light of (35), which agrees perfectly with Fisher's $(1930,1941)$ writings about the fundamental theorem of natural selection except that time is discrete and generations are nonoverlapping.

\section{DISCRETE-TIME MODEL WITH OVERLAPPING GENERATIONS}

\subsection{Model without Age Effects}

Let time be divided into nonoverlapping intervals of length $\Delta t$. During such a time interval, individuals whose genotype is $g$ irrespective of their age produce a mean number of offspring equal to $\varphi^{(g)}$, counting half an offspring for each successful gamete. At the end of the time interval, the individuals die or survive. Let the probability of surviving for an additional time period $\Delta t$ be $s^{(g)}$ for individuals of genotype $g$, whatever their age may be. An individual who survives can be considered as being produced by itself and as carrying two successful gametes. With this convention, the parameter

$$
w^{(g)}=\varphi^{(g)}+s^{(g)}
$$


represents the mean number of individuals at the beginning of the next time period $\Delta t$ produced by individuals of genotype $g$ at the beginning of the current time period $\Delta t$.

Similarly, if $\Phi^{(g)}$ is the mean number of $g$ offspring among all offspring at the beginning of the next time period $\Delta t$ per $g$ individual in the whole population at the beginning of the current time period $\Delta t$, then

$$
W^{(g)}=\Phi^{(g)}+s^{(g)}
$$

is the mean number of individuals of genotype $g$ including those who survive at the beginning of the next time period $\Delta t$ per individual of genotype $g$ at the beginning of the current time period $\Delta t$.

The conclusions of the previous sections remain fully valid with these genotypic fitnesses $w^{(g)}$ and genotypic growth rates $W^{(g)}$ if we substitute "current time interval" for "current generation" and "next time interval" for "next generation."

\subsection{Model with Age Effects}

If the selection parameters, namely, the viability parameters, as well as the fecundity parameters, depend on age, then we have recourse to the notion of reproductive value. Let $\varphi_{k}^{(g)}$ and $s_{k}^{(g)}$ be the mean number of offspring and the survival probability, respectively, both over a time period $\Delta t$, for individuals of genotype $g$ in age class $((k-1) \Delta t, k \Delta t]$, represented by $k$, for $k \geqslant 1$. Let $w^{(g)}$ be the unique positive solution of the equation

$$
\sum_{k \geqslant 1} \frac{L_{k}^{(g)} \varphi_{k}^{(g)}}{\left[w^{(g)}\right]^{k}}=1,
$$

where $L_{k}^{(g)}$ is the probability that an individual of genotype $g$ reaches age class $k$, that is,

$$
L_{k}^{(g)}=s_{1}^{(g)} \times \cdots \times s_{k-1}^{(g)}
$$

for $k \geqslant 2$ and $L_{1}^{(g)}=1$. Then the reproductive value of the individuals of genotype $g$ in age class $k$ is defined as

$$
v_{k}^{(g)}=\frac{\left[w^{(g)}\right]^{k-1}}{L_{k}^{(g)}} \sum_{l \geqslant k} \frac{L_{l}^{(g)} \varphi_{l}^{(g)}}{\left[w^{(g)}\right]^{l}}
$$

for $k \geqslant 2$ and $v_{1}^{(g)}=1$. It can be checked that the reproductive value satisfies the equation

$$
\varphi_{k}^{(g)}+s_{k}^{(g)} v_{k+1}^{(g)}=w^{(g)} v_{k}^{(g)} .
$$

The left-side member of this equation represents the mean reproductive value of the individuals in the population at the beginning of the next time period $\Delta t$ that are produced by the individuals of genotype $g$ in age class $k$ in the population at the beginning of the current time period $\Delta t$, if we assume constant reproductive values from the current time period $\Delta t$ to the next time period $\Delta t$. (This will be the case if the parameters $\varphi_{k}^{(g)}$ and $s_{k}^{(g)}$ remain constant.) The equality of this with the right-side member of (41) means that $w^{(g)}$ is the mean amount of reproductive value in the population at the beginning of the next time period $\Delta t$ that is produced, per unit of reproductive value, by individuals of genotype $g$ at the beginning of the current time period $\Delta t$ irrespective of their age. This is to be used as the definition of genotypic fitness.

The parameter $w^{(g)}$ corresponds to the growth rate of every age class with respect to $g$ individuals if the frequency of age class $k$ among $g$ individuals is proportional to $L_{k}^{(g)} /\left[w^{(g)}\right]^{k-1}$, which defines a steady age-distribution for $g$ individuals. The growth rate of age class $k$ with respect to $g$ individuals is interpreted as the ratio, in numbers, of individuals in age class $k$ at the beginning of the next time period $\Delta t$ produced by $g$ individuals, to individuals of genotype $g$ in age class $k$ at the beginning of the current time period $\Delta t$. On the other hand, the reproductive value is interpreted as the contribution "to the ancestry of future generations" (Fisher, 1958, p. 27). Note that, if the selection parameters do not depend on age as in the previous subsection, then we get $v_{k}^{(g)}=1$ for every $k \geqslant 1$ and $w^{(g)}=\varphi^{(g)}+s^{(g)}$.

Under the assumption of constant reproductive values, let $x_{k}^{(g)}$ be the frequency of individuals of genotype $g$ in age class $k$ counted as many times as their reproductive value and $x_{k}^{(g) *}$ be the frequency of the same individuals counted as many times as the mean reproductive value of individuals in the population at the beginning of the next time interval that are produced by them. Then we have

$$
x_{k}^{(g) *}=\frac{x_{k}^{(g)} w^{(g)}}{\bar{w}},
$$

where

$$
\bar{w}=\sum_{g} \sum_{k \geqslant 1} x_{k}^{(g)} w^{(g)} .
$$

It suffices to define

$$
x^{(g)}=\sum_{k \geqslant 1} x_{k}^{(g)}
$$


and

$$
m^{(g)}=b^{(g)}-d^{(g)}
$$

$$
x^{(g)^{*}}=\sum_{k \geqslant 1} x_{k}^{(g)^{*}}
$$

for the conclusions of the previous section to apply, mutatis mutandis, with "current generation" replaced by "current time interval" and "next generation" by "next time interval."

Note that, in this case, the growth rate of genotype $g$ takes the form

$$
W^{(g)}=\frac{1}{x^{(g)}} \sum_{k \geqslant 1}\left[\frac{\Phi_{k}^{(g)}+s_{k}^{(g)} v_{k+1}^{(g)}}{v_{k}^{(g)}}\right] x_{k}^{(g)},
$$

where $\Phi_{k}^{(g)}$ represents the mean number of $g$ offspring, among all offspring at the beginning of the next time period $\Delta t$, per $g$ individual in age class $k$ at the beginning of the current time period $\Delta t$.

\section{CONTINUOUS-TIME MODEL WITH OVERLAPPING GENERATIONS}

\subsection{Model without Age Effects}

The continuous-time model without age effects is obtained by setting

$$
\begin{aligned}
& \varphi^{(g)}=b^{(g)} \Delta t+o(\Delta t), \\
& \Phi^{(g)}=B^{(g)} \Delta t+o(\Delta t), \\
& s^{(g)}=1-d^{(g)} \Delta t+o(\Delta t)
\end{aligned}
$$

(where $o(\Delta t)$ denotes any function of $\Delta t$ such that $o(\Delta t) / \Delta t$ goes to 0 as $\Delta t$ goes to 0$)$ in the corresponding discrete-time model with overlapping generations and by letting $\Delta t$ go to 0 . The parameters $b^{(g)}, B^{(g)}$, and $d^{(g)}$ are respectively the birth rate (the rate at which $g$ individuals give birth), the born rate (the rate at which $g$ individuals are born; Denniston, 1978), and the death rate (the rate at which $g$ individuals die). Then the fitness and the growth rate of genotype $g$ take the forms

$$
\begin{gathered}
w^{(g)}=1+m^{(g)} \Delta t+o(\Delta t), \\
W^{(g)}=1+M^{(g)} \Delta t+o(\Delta t),
\end{gathered}
$$

where is the Malthusian parameter (Fisher, 1958, p. 26) for genotype $g$, and

$$
M^{(g)}=B^{(g)}-d^{(g)}
$$

is the relative rate of change in the frequency of genotype $g$, that is, the derivative with respect to time of the natural logarithm of $x^{(g)}$. The corresponding additive value and residual addends are in the form

$$
\begin{aligned}
& \alpha^{(g)}=\eta^{(g)} \Delta t+o(\Delta t), \\
& \delta^{(g)}=\zeta^{(g)} \Delta t+o(\Delta t), \\
& \varepsilon^{(g)}=\tau^{(g)} \Delta t+o(\Delta t),
\end{aligned}
$$

where $\eta^{(g)}$ is the additive value of both $m^{(g)}$ and $M^{(g)}$, while $\zeta^{(g)}$ and $\tau^{(g)}$ are their respective residual addends. Then, letting $\Delta t$ go to 0 , we get, for the frequency of allele $A_{i}$,

$$
\frac{d p_{i}}{d t}=\operatorname{cov}\left(f_{i}, m\right)=\operatorname{cov}\left(f_{i}, M\right)=\operatorname{cov}\left(f_{i}, \eta\right)
$$

and, for the mean Malthusian parameter,

$$
\frac{d \bar{m}}{d t}=\sigma_{\eta}^{2}+\operatorname{cov}(\zeta, \tau)+\frac{\overline{d m}}{d t},
$$

where

$$
\frac{\overline{d m}}{d t}=\sum_{g} x^{(g)} \frac{d m^{(g)}}{d t}
$$

and

$$
\sigma_{\eta}^{2}=\sum_{g}\left[\frac{d x^{(g)}}{d t}\right]_{\eta} m^{(g)}
$$

with

$$
\left[\frac{d x^{(g)}}{d t}\right]_{\eta}=x^{(g)} \eta^{(g)} .
$$

Equation (43) corresponds to Kimura's (1958) decomposition for the mean Malthusian parameter if an appropriate change of variables is made (see Section 8.2 for details). Equation (44) specifies the interpretation to give to the first term in this decomposition in order to be in agreement with Fisher's (1930) FTNS. 


\subsection{Model with Age Effects}

For individuals of genotype $g$ in age class $k$, let

$$
\varphi_{k \Delta t}^{(g)}=b_{k \Delta t}^{(g)} \Delta t+o(\Delta t)
$$

and

$$
s_{k \Delta t}^{(g)}=1-d_{k \Delta t}^{(g)} \Delta t+o(\Delta t)
$$

be respectively the mean number of offspring from age $(k-1) \Delta t$ to age $k \Delta t$ and the probability of living for an additional time period $\Delta t$ from age $k \Delta t$. Then the fitness of genotype $g$ is in the form

$$
w^{(g)}=1+m^{(g)} \Delta t+o(\Delta t),
$$

where $m^{(g)}$ is the corresponding Malthusian parameter (Fisher, 1958, p. 26), which satisfies the analogue of (39) in continuous time

$$
\int_{0}^{\infty} e^{-m^{(g)} t} L_{t}^{(g)} \varphi_{t}^{(g)} d t=1
$$

with

$$
L_{u}^{(g)}=e^{-\int_{0}^{\infty} d_{t}^{(g)} d t}
$$

that is,

$$
d_{u}^{(g)}=-\frac{d}{d u} \log L_{u}^{(g)},
$$

where $\varphi_{u}^{(g)}$ and $d_{u}^{(g)}$ are limits of $\varphi_{k \Delta t}^{(g)}$ and $d_{k \Delta t}^{(g)}$, respectively, as $\Delta t$ goes to 0 and $k$ goes to $\infty$ such that $k \Delta t$ goes to $u$. In this case, the reproductive value of individuals of genotype $g$ at age $u$ is

$$
v_{u}^{(g)}=\frac{e^{m^{(g)} u}}{L_{u}^{(g)}} \int_{u}^{\infty} e^{-m^{(g)} t} L_{t}^{(g)} \varphi_{t}^{(g)} d t
$$

and satisfies the equation

$$
m^{(g)} v_{u}^{(g)} d u=d v_{u}^{(g)}+\left(b_{u}^{(g)}-d_{u}^{(g)} v_{u}^{(g)}\right) d u,
$$

which are the analogues of (40) and (41) in continuous time given by Fisher (1958, pp. 27 and 30).

The parameter $m^{(g)}$, which is the real solution to Lotka's (1922) Eq. (45), is also known as the intrinsic rate of increase of genotype $g$ (Norton, 1928). It must be distinguished from the relative (geometric) rate of change in the frequency of genotype $g$, which would correspond to an unconventional definition of fitness (Denniston, 1978), although this interpretation has been occasionally given to Fisher's (1930) concept of the Malthusian parameter (see, e.g., Charlesworth, 1970).

The previous results in continuous time apply if individuals are weighted according to their reproductive value and if the reproductive values are assumed constant in time. This agrees, using different variables, with Crow's (1979) analysis, which extends Kimura's (1958) formula for one-locus models to age-structured populations.

\section{DISCUSSION}

\subsection{Partial Change in Mean Fitness}

The increase in fitness meant by Fisher (1930) in his statement of the FTNS might be, in a discrete-time model with nonoverlapping generations, the partial change

$$
\sum_{g}\left[\Delta x^{(g)}\right]_{\alpha} w^{(g)},
$$

where $\left[\Delta x^{(g)}\right]_{\alpha}$ represents the change in the frequency of genotype $g$ ascribable only to changes in gene frequencies and $w^{(g)}$ is the fitness of $g$. This is suggested by Fisher's (1941) own explanations. For the change in mean fitness to be the sum of average effects of genes times their change in frequency without restrictive assumptions on genetic determination, which is the key for the derivation of the FTNS, the environment, including the genic environment, has to be kept constant. This means constant genotypic fitnesses and changes in genotypic frequencies directly consequent on changes in gene frequencies. With fitness determined by two alleles at a single autosomal locus in a diploid population, this is the case when the relative change in the frequency of an ordered heterozygote is the average of the relative changes in the frequencies of the two homozygotes (Eq. (1) in continuous time according to Fisher, 1941, and Eq. (3) in discrete time according to Kempthorne, 1957). This occurs (exactly in continuous time, approximately in discrete time) when the ratio $\lambda$ of the square of the frequency of the ordered heterozygote and the product of the frequencies of both homozygotes remains constant (see Eq. (2)). This ratio is identically equal to 1 if the frequencies of the ordered genotypes at the single autosomal 
locus are products of the frequencies of the genes present at that locus, which is to say that Hardy-Weinberg proportions hold for the genotypic frequencies.

A generalization of condition (3) to ensure a constant genic environment with any number of loci and any number of alleles at each locus is

$$
\Delta x^{(g)}=\left[\Delta x^{(g)}\right]_{\alpha}
$$

with

$$
\left[\Delta x^{(g)}\right]_{\alpha}=\frac{2 x^{(g)}}{\bar{w}} \sum_{i} f_{i}^{(g)} \alpha_{i},
$$

where $x^{(g)}$ is the frequency of an ordered genotype $g$ at the $L$ loci, $f_{i}^{(g)}$ is the frequency of allele $A_{i}$ in $g, \alpha_{i}$ is the additive effect of allele $A_{i}$ on fitness, and $\bar{w}$ is the mean fitness. Then the relative changes in genotypic frequencies are linear with respect to gene frequencies in genotype. This is the case (exactly in continuous time, approximately in discrete time), with $\alpha_{i}$ given by $\bar{w} \Delta p_{i} / p_{i}$ or $(\bar{w} / 2) \Delta P_{i i} / P_{i i}$, where $p_{i}$ is the frequency of $A_{i}$ and $P_{i i}$ is the frequency of $A_{i} A_{i}$, when the frequencies of the ordered genotypes at the $L$ loci are the products of the frequencies of the maternal and paternal genes present at the $L$ loci, namely, when the genotypes at the $L$ loci are in Hardy-Weinberg-Robbins proportions. Then the gametes at the $L$ loci are in Robbins proportions, which means that there is linkage equilibrium over loci, and the genotypes at each of the $L$ loci are in Hardy-Weinberg proportions.

But, in general, condition (49) is not satisfied. Nevertheless, we have always, using (8), (10), and (16), the identity

$$
\sum_{g}\left[\Delta x^{(g)}\right]_{\alpha} w^{(g)}=2 \sum_{i}\left(\Delta p_{i}\right) \alpha_{i},
$$

from which we get, owing to (27), the equation

$$
\sum_{g}\left[\Delta x^{(g)}\right]_{\alpha} w^{(g)}=\frac{\sigma_{\alpha}^{2}}{\bar{w}},
$$

where $\sigma_{\alpha}^{2}$ is the additive genetic variance in fitness and $\bar{w}$ is the mean fitness. Since $\left[\Delta x^{(g)}\right]_{\alpha}$ is only a partial change in the frequency of genotype $g$, its change as best predicted from the changes in gene frequencies, the above expression represents a partial change in mean fitness even in the case of constant genotypic fitnesses. The equality of this partial change to the additive genetic variance in fitness divided by the mean fitness is the discrete-time version of Fisher's FTNS.
It is important to note that the equality (50) holds essentially because the genotypic growth rate defined by (6) has the same average excesses and therefore the same average effects with respect to gene substitutions as the genotypic fitness has. This is essentially because both concepts coincide for genes: the allelic fitnesses are the same as the allelic growth rates. It is ironic that Denniston's (1978) study on the genotypic growth rate as an incorrect definition of genotypic fitness turns out to be precursory to a correct interpretation of the FTNS.

Result (50) is valid for discrete-time models with overlapping generations with or without age effects on viability and fecundity if individuals are "weighted according to the reproductive value of each" (Fisher, 1958 , p. 38) and if fitness is defined as the contribution to the growth rate of the population in reproductive value. Without age effects on viability and fecundity, all reproductive values are equal to 1 and fitness corresponds to the contribution to the growth rate in the total number of individuals in the population. With age effects on viability and fecundity, reproductive values depend on age and genotype but are kept constant in the partial change (50) under constant environment, since constant environment must include constant viability and fecundity parameters, which imply constant reproductive values as well as constant fitnesses according to (39) and (40). This is the condition for the basic equation (41) used to interpret fitness to be valid.

The analogue of (50) in continuous time is

$$
\sum_{g}\left[\frac{d x^{(g)}}{d t}\right]_{\eta} m^{(g)}=\sigma_{\eta}^{2},
$$

where $m^{(g)}$ is the Malthusian parameter for genotype $g$ as defined by Fisher $(1958$, p. 26$),\left[d x^{(g)} / d t\right]_{\eta}$ is the rate of change in $x^{(g)}$ ascribable only to changes in gene frequencies, everything else being kept constant, including fitnesses and reproductive values characterized by (45), (46), and (47), and $\sigma_{\eta}^{2}$ is the additive genetic variance in the Malthusian parameter. This is exactly Fisher's FTNS.

That the FTNS concerns a partial change in mean fitness has already been put forward by Price (1972) for continuous-time models and by Ewens (1989) for discrete-time as well as continuous-time models. In our notation and in a discrete-time model with nonoverlapping generations, their expression for that partial change is

$$
\sum_{g}\left[\Delta x^{(g)}\right] w_{\alpha}^{(g)},
$$


where

$$
w_{\alpha}^{(g)}=\bar{w}+2 \sum_{i} f_{i}^{(g)} \alpha_{i}
$$

is the fitness of genotype $g$ as best predicted from the genes in $g$. We get immediately from (8)

$$
\sum_{g}\left[\Delta x^{(g)}\right] w_{\alpha}^{(g)}=2 \sum_{i}\left(\Delta p_{i}\right) \alpha_{i}
$$

from which

$$
\sum_{g}\left[\Delta x^{(g)}\right] w_{\alpha}^{(g)}=\frac{\sigma_{\alpha}^{2}}{\bar{w}},
$$

owing to (27) (see also Ewens, 1992, and Castilloux and Lessard, 1995). The analogue in continuous time in the notation of Section 7 is

$$
\sum_{g}\left[\frac{d x^{(g)}}{d t}\right] m_{\eta}^{(g)}=\sigma_{\eta}^{2},
$$

where $m_{\eta}^{(g)}$ is the Malthusian parameter of $g$ as best predicted from the genes present in $g$ and $\sigma_{\eta}^{2}$ is the additive genetic variance in Malthusian parameter.

But as Price (1972) points out, Fisher (1958) speaks of "the value of the genotype as best predicted from the genes present" (p. 35) as "the genetic value" (p. 36) and uses the notation $\xi$ for this value (which corresponds to $w_{\alpha}^{(g)}-\bar{w}$ in discrete time and $m_{\eta}^{(g)}-\bar{m}$ in continuous time in our notation), while in "no case does he use this notation or this terminology in connection with the "fitness" mentioned in his theorem" (Price, 1972, p. 133). Moreover, even if the partial change in (51) is equal to the additive genetic variance in fitness divided by the mean fitness in accordance with a dicrete-time version of the FTNS, "this does not mean that Fisher would have accepted [(52)] as a statement of his theorem" (Price, 1972, p. 133). Of course, the same could be said about our partial change (48), but this partial change is suggested by Fisher's (1941) own condition on the change in genotypic frequencies, given in (1), for the change in mean fitness to be ascribable only to changes in gene frequencies in the case of constant genotypic fitnesses. Nevertheless, it is surprising that Fisher did not include this explanation on the FTNS in the 1958 edition of his book "The Genetical Theory of Natural Selection" as if it would not be necessary. This would be so if natural selection concerned changes due only to changes in gene frequencies, the genes being the units of selection, and average effects of genes were defined as rates of changes due only to changes in gene frequencies. Therefore, Fisher's (1941) paper would not be about the FTNS but about the notion of average effect as suggested by the title of his paper.

Another discrepancy between our results and Price's (1972) results is found in the definition of fitness in the case of overlapping generations with age effects on viability and fecundity. While Price (1972) sees an "inconsistency in Fisher's mathematics" (Price and Smith, 1972, p. 3 ) and proposes a definition of fitness that takes into account changes in viability and fecundity parameters, we believe that Fisher's definition of fitness is perfectly consistent with his views, since such changes belong to changes in the environment and, as a consequence, they must be ignored in the FTNS.

In the case of nonoverlapping generations, a definition of fitness in agreement with Fisher's views incorporates fecundity as well as viability differences. This is the case with fitness defined as the mean number of offspring from the beginning to the end of the current generation. Such a fitness for genotypes is more general than viability selection used by Ewens (1989) for his interpretation of the FTNS in discrete time, but it is more likely to be frequency-dependent since fecundities are based on mating types. As a matter of fact, unless fecundities of mating types are products of constant factors that depend on the male and female genotypes, marginal fecundities associated with genotypes will be frequency-dependent. Moreover, with our more general definition of fitness, the assumption that mating and reproduction do not change gene frequencies from the current generation at the time of maturity to the next generation at the time of conception is replaced by the much weaker assumptions of Mendelian segregation and no gametic selection. This has some importance since several nonrandom mating patterns change gene frequencies and these are not excluded in Fisher's FTNS.

Note that fecundity selection can also be treated using mating types, instead of genotypes, as units of selection. The FTNS in Ewens' sense has been asserted in this context too (Lessard and Castilloux, 1995), but under the assumption that mating does not change gene frequencies from the time of conception to the time of reproduction. Again this is rather restrictive for nonrandom mating populations. But the partial change in the mean fecundity obtained with this approach should explain a greater part of the total change, since, with fecundity differences, the marginal genotypic fitnesses are most likely to be frequency-dependent even if the fecundity parameters are not. 


\subsection{Total Change in Mean Fitness}

From Eqs. (13), (23), (28), (30), and (38), the total change in mean fitness in a discrete-time model with nonoverlapping generations can be expressed as

$$
\Delta \bar{w}=\frac{\operatorname{cov}(W, w)}{\bar{w}}+\overline{\Delta w}+\frac{\operatorname{cov}(W, \Delta w)}{\bar{w}},
$$

where

$$
\operatorname{cov}(W, w)=\sigma_{\alpha}^{2}+\operatorname{cov}(\varepsilon, \delta)
$$

and

$$
\operatorname{cov}(W, \Delta w)=\operatorname{cov}(\alpha, \Delta w)+\operatorname{cov}(\varepsilon, \Delta w) .
$$

Therefore, even in the case of constant genotypic fitnesses (which means $\Delta w=0$ ), the total change in mean fitness involves a second term, besides the additive genetic variance in fitness divided by the mean fitness, which depends on residual addends for dominance and epistatic effects of genes. More precisely, this term is the covariance between the residual addend of the genotypic fitness given by

$$
\delta^{(g)}=w^{(g)}-\bar{w}-\alpha^{(g)}
$$

and the residual addend of the genotypic growth rate given by

$$
\varepsilon^{(g)}=W^{(g)}-\bar{W}-\alpha^{(g)},
$$

divided by the mean fitness $\bar{w}$. This result is essentially based on decomposition (33) for the change in genotype frequencies, which comes from decomposition (28) for the genotypic growth rate minus its mean, by the leastsquares method, into the additive fitness and a residual addend.

By contrast, in a discrete-time analogous version and in our notation, Kimura's (1958) formula is essentially based on the decomposition for the genotypic growth rate,

$$
W^{(g)}-\bar{W}=a^{(g)}+e^{(g)},
$$

where

$$
a^{(g)}=2 \sum_{i} f_{i}^{(g)} a_{i}
$$

and

$$
e^{(g)}=W^{(g)}-\bar{W}-a^{(g)} .
$$

The quantity $a_{i}$ in the definition of $a^{(g)}$ is the average excess in fitness of allele $A_{i}$, given by $\bar{w} \Delta p_{i} / p_{i}$ according to (12), which is usually different from the additive effect of allele $A_{i}$, denoted by $\alpha_{i}$, and therefore $a^{(g)}$ is usually different from the additive fitness $\alpha^{(g)}$ defined in (14). Then, schematically, the analysis goes as follows:

$$
\begin{aligned}
\operatorname{cov}(W, w) & =\operatorname{cov}(a, w)+\operatorname{cov}(e, w) \\
& =\operatorname{cov}(a, \alpha)+\operatorname{cov}(a, \delta)+\operatorname{cov}(e, w) \\
& =\operatorname{cov}(W, \alpha)-\operatorname{cov}(e, \alpha)+\operatorname{cov}(e, w) \\
& =\operatorname{cov}(W, \alpha)+\operatorname{cov}(e, \delta) \\
& =\sigma_{\alpha}^{2}+\operatorname{cov}(e, \delta),
\end{aligned}
$$

since, owing to (20),

$$
\operatorname{cov}(a, \delta)=2 \sum_{i} \operatorname{cov}\left(f_{i}, \delta\right) a_{i}=0,
$$

while, owing to (27),

$$
\begin{aligned}
\operatorname{cov}(W, \alpha) & =\bar{w} \sum_{g} x^{(g)}\left[\frac{\Delta x^{(g)}}{x^{(g)}}\right] \alpha^{(g)} \\
& =2 \bar{w} \sum_{i} \sum_{g}\left[\Delta x^{(g)}\right] f_{i}^{(g)} \alpha_{i} \\
& =2 \bar{w} \sum_{i}\left[\Delta p_{i}\right] \alpha_{i}=\sigma_{\alpha}^{2} .
\end{aligned}
$$

This analysis does not use the property that the genotypic growth rate shares the same additive value as the genotypic fitness.

Comparing (55) and (57), or using directly (20), we get immediately

$$
\frac{\operatorname{cov}(\varepsilon, \delta)}{\bar{w}}=\frac{\operatorname{cov}(e, \delta)}{\bar{w}},
$$

from which

$$
\frac{\operatorname{cov}(\varepsilon, \delta)}{\bar{w}}=\sum_{g} x^{(g)}\left[\frac{\Delta x^{(g)}}{x^{(g)}}-2 \sum_{i} f_{i}^{(g)} \frac{\Delta p_{i}}{p_{i}}\right] \delta^{(g)} .
$$

In the case of a single locus, this becomes

$$
\frac{\operatorname{cov}(\varepsilon, \delta)}{\bar{w}}=\sum_{i j} P_{i j}\left[\frac{\Delta P_{i j}}{P_{i j}}-\frac{\Delta p_{i}}{p_{i}}-\frac{\Delta p_{j}}{p_{j}}\right] \delta_{i j},
$$


where $P_{i j}$ is the frequency of genotype $A_{i} A_{j}$ and $\delta_{i j}$ is the dominance deviation in fitness of this genotype. If selection is weak so that changes in genotypic and allelic frequencies are small, then the term in square brackets in (60) is approximately equal to $\Delta \theta_{i j} / \theta_{i j}$, which is approximately equal to $\Delta\left(\log \theta_{i j}\right)$, where

$$
\theta_{i j}=\frac{P_{i j}}{p_{i} p_{j}}
$$

is the coefficient of departure from random mating as defined by Kimura (1958).

The continuous-time analogue of $(60)$ in the notation of Section 7 is

$$
\operatorname{cov}(\zeta, \tau)=\sum_{i j} P_{i j} \frac{d}{d t}\left(\log \theta_{i j}\right) \zeta_{i j},
$$

and then Eq. (42), in the case of a single locus, corresponds exactly to Kimura's (1958) formula for the total rate of change in the mean Malthusian parameter. Equation (61) has been deduced by Denniston (1978) for any quantitative character $c$ having residual addend $\zeta$, determined at a single locus and correlated with the relative rate of change in the frequencies of the corresponding genotypes, represented here by $M$ and having residual addend $\tau$, from the equation

$$
\frac{d \bar{c}}{d t}=\operatorname{cov}(M, c)+\frac{\overline{d c}}{d t}
$$

This equation for the total rate of change in the mean of a quantitative character is not limited to one-locus models and a discrete-time version is possible, as claimed by Denniston and as confirmed by Eqs. (42) and (54) in the case where the quantitative character is fitness. Equations (54) and (62) are actually valid for any quantitative character determined at any number of loci and lead to an interpretation of Robertson's (1966) secondary theorem of natural selection (STNS) if the character is kept constant and the genotypic growth rate $W$ in discrete time or the relative rate of change in genotype frequencies $M$ in continuous time is replaced by the corresponding additive value. This partial change in the mean character is the change ascribable only to changes in gene frequencies and is equal to the covariance between the additive value of the character and the additive value of fitness, divided by the mean value of the character if time is discrete. For more details and references on the STNS, see, e.g., Crow and Nagylaki (1976), and Nagylaki (1989, 1991, 1993).
Similarly to (56), if we use the decomposition

$$
W^{(g)}-\bar{W}=H^{(g)}+E^{(g)},
$$

where

$$
H^{(g)}=\bar{w} \sum_{i} f_{i}^{(g)} \frac{\Delta P_{i i}}{P_{i i}}
$$

and

$$
E^{(g)}=W^{(g)}-\bar{W}-H^{(g)},
$$

then we get

$$
\begin{aligned}
\frac{\operatorname{cov}(\varepsilon, \delta)}{\bar{w}} & =\frac{\operatorname{cov}(E, \delta)}{\bar{w}} \\
& =\sum_{g} x^{(g)}\left[\frac{\Delta x^{(g)}}{x^{(g)}}-\sum_{i} f_{i}^{(g)} \frac{\Delta P_{i i}}{P_{i i}}\right] \delta^{(g)} .
\end{aligned}
$$

In the case of a single locus, this becomes

$$
\begin{aligned}
& \frac{\operatorname{cov}(\varepsilon, \delta)}{\bar{w}} \\
& \quad=\frac{1}{2} \sum_{i} \sum_{j \neq i} P_{i j}\left[\frac{2 \Delta P_{i j}}{P_{i j}}-\frac{\Delta P_{i i}}{P_{i i}}-\frac{\Delta P_{j j}}{P_{j j}}\right] \delta_{i j} .
\end{aligned}
$$

If selection is weak, then the term in square brackets in (63) is approximately equal to $\Delta \lambda_{i j} / \lambda_{i j}$ with

$$
\lambda_{i j}=\frac{P_{i j}^{2}}{P_{i i} P_{j j}},
$$

as in Kempthorne's (1957, p. 361) approximate formula for the total change in mean fitness in discrete time.

Equations (60) and (63) under weak selection are related by the equality

$$
\lambda_{i j}=\frac{\theta_{i j}^{2}}{\theta_{i i} \theta_{j j}},
$$

from which we have the approximation

$$
\frac{\Delta \lambda_{i j}}{\lambda_{i j}} \simeq 2 \frac{\Delta \theta_{i j}}{\theta_{i j}}-\frac{\Delta \theta_{i i}}{\theta_{i i}}-\frac{\Delta \theta_{j j}}{\theta_{j j}} .
$$

Using the fact that

$$
\sum_{j \neq i} P_{i j} \delta_{i j}=-P_{i i} \delta_{i i}
$$


which comes from (20), we get

$$
\frac{1}{2} \sum_{i} \sum_{j \neq i} P_{i j} \frac{\Delta \lambda_{i j}}{\lambda_{i j}} \delta_{i j} \simeq \sum_{i j} P_{i j} \frac{\Delta \theta_{i j}}{\theta_{i j}} \delta_{i j} .
$$

This term in the total change of mean fitness is attributed by Kempthorne (1957) to changes in the mating system. Kimura (1958) presents the continuous-time analogue (61), extends the analysis to the case of two loci, and sketches the result for multiple loci. However, in this case his decomposition over loci is incomplete as pointed out by Nagylaki (1989), who gives the exact expression in the case of two loci.

We have shown that Kempthorne's (1957) and Kimura's (1958) decompositions of the total change in mean fitness are two different forms of the same basic decomposition obtained by the least-squares method applied not only to the genotypic fitness but also to the genotypic growth rate. This is a more natural decomposition from a statistical point of view, which enlightens and unifies the previous ones. These are based on a decomposition of the genotypic growth rate into a linear function with respect to gene frequencies in genotype and a remaining term as in (56). The linear function has been chosen by Kempthorne and by Kimura in such a way as to have a genetic interpretation. But this is not necessary and we can imagine many other decompositions such as the one in (28) where the linear function is the additive value obtained by least squares. This approach is particularly appealing and should facilitate further decompositions.

It is worth noting that, if selection is weak enough and if mating is random, then genotypic frequencies approach Hardy-Weinberg-Robbins proportions and the term in the total change of mean fitness due to changes in the mating system becomes negligible, in the sense that it is of higher order with respect to selection intensity, compared to the term due only to changes in gene frequencies, at least after enough time has passed, as shown by Nagylaki (1993) for a discrete-time model with nonoverlapping generations. Then the total change in mean fitness is approximately given by the additive genetic variance in fitness divided by the mean fitness as long as the gene-frequency changes remain of lower order with respect to selection intensity, which should be the case in a population sufficiently far from equilibrium, unless symmetry conditions are satisfied. This result has been called the asymptotic fundamental theorem of natural selection as opposed to the Fisher-Price-Ewens theorem of natural selection, which says that a partial change in mean fitness is exactly equal to the additive genetic variance in fitness divided by the mean fitness. The approximation is valid even in the case of genotypic fitnesses depending on gametic frequencies and time as long as their changes are of smaller order compared to selection intensity. However, the assumptions of weak selection and random mating remain crucial.

In general, when the genotypic fitness parameters vary with respect to time, the total change in mean fitness includes terms that depend on changes in these parameters. The first is the mean of changes in genotypic fitness while the second is the covariance of the genotypic growth rate and the change in genotypic fitness divided by the mean fitness (see Eq. (54)). In continuous time, the second disappears (see Eq. (62)).

It is important to stress that our decomposition of the total change in mean fitness in discrete time as well as in continuous time applies to models with age effects in populations with overlapping generations, but is exact only under the assumption of constant reproductive values, which almost implies constant viabilities and fecundities. For an asymptotic analysis in this case with constant and small differences in viabilities and fecundities, we refer the reader to Charlesworth (1974). (See also Charlesworth, 1980, for more details on agestructured populations.)

If reproductive values in age-structured populations are not constant, then the total change in mean fitness includes supplementary terms. But the FTNS does not concern these terms and our interpretation of the theorem remains fully valid in this context which is Fisher's original framework.

\section{ACKNOWLEDGMENTS}

I am grateful to Professor Warren J. Ewens for very helpful comments.

\section{REFERENCES}

Castilloux, A.-M., and Lessard, S. 1995. The fundamental theorem of natural selection in Ewens' sense (Case of many loci), Theor. Popul. Biol. 48, 306-315.

Charlesworth, B. 1970. Selection in populations with overlapping generations. I. The use of Malthusian parameters in population genetics, Theor. Popul. Biol. 1, 352-370.

Charlesworth, B. 1974. Selection in populations with overlapping generations. VI. Rates of change of gene frequency and population growth rate, Theor. Popul. Biol. 6, 108-133.

Charlesworth, B. 1980. "Evolution in Age-Structured Populations," Cambridge Univ. Press, Cambridge.

Crow, J. F. 1979. Gene frequency and fitness change in an age-structured population, Ann. Hum. Genet. 42, 355-370. 
Crow, J. F., and Nagylaki, T. 1976. The rate of change of a character correlated with fitness, Am. Nat. 110, 207-213.

Darwin, C. 1956. "On the Origin of Species by Means of Natural Selection, or the Preservation of Favoured Races in the Struggle for Life," reprint of the 6th ed., Oxford Univ. Press, London.

Denniston, C. 1978. An incorrect defintion of fitness revisited, Ann. Hum. Genet. 42, 77-85.

Edwards, A. W. F. 1994. The fundamental theorem of natural selection, Biol. Rev. 69, 443-474.

Emlen, J. M. 1970. Age specificity and ecological theory, Ecology 51, 588-601.

Ewens, W. J. 1989. An interpretation and proof of the fundamental theorem of natural selection, Theor. Popul. Biol. 36, 167-180.

Ewens, W. J. 1992. An optimizing principle of natural selection in evolutionary population genetics, Theor. Popul. Biol. 42, 333-346.

Fisher, R. A. 1930. "The Genetical Theory of Natural Selection," Clarendon, Oxford.

Fisher, R. A. 1941. Average excess and average effect of a gene substitution, Ann. Eugenics 11, 53-63.

Fisher, R. A. 1958. "The Genetical Theory of Natural Selection," 2nd rev. ed., Dover, New York.

Frank, S. A., and Slatkin, M. 1992. Fisher's fundamental theorem of natural selection, Trends Ecol. Evol. 7, 92-95.

Kempthorne, O. 1957. "An Introduction to Genetic Statistics," Wiley, New York.

Kimura, M. 1956. A model of a genetic system which leads to closer linkage by natural selection, Evolution 10, 278-287.

Kimura, M. 1958. On the change of population fitness by natural selection, Heredity 12, 145-167.
Lessard, S., and Castilloux, A.-M. 1995. The fundamental theorem of natural selection in Ewens' sense (Case of fertility selection), Genetics 141, 733-742.

Li, C. C. 1955. "Population Genetics," Chicago Univ. Press, Chicago.

Li, C. C. 1967. Fundamental theorem of natural selection, Nature 214, 505-506.

Li, C. C. 1969. Increment of average fitness for multiple alleles, Proc. Natl. Acad. Sci. U.S.A. 62, 395-398.

Lotka, A. J. 1922. The stability of the normal age distribution, Proc. Natl. Acad. Sci U.S.A. 8, 339-345.

Nagylaki, T. 1989. Rate of evolution of a character without epistasis, Proc. Natl. Acad. Sci. U.S.A. 86, 1910-1913.

Nagylaki, T. 1991. Error bounds for the fundamental and secondary theorems of natural selection, Proc. Natl. Acad. Sci. U.S.A. 88, 2402-2406.

Nagylaki, T. 1993. The evolution of multilocus systems under weak selection, Genetics 134, 627-647.

Norton, H. T. J. 1928. Natural selection and Mendelian variation, Proc. London Math. Soc. 28, 1-45.

Price, G. R. 1970. Selection and covariance, Nature 227, 520-521.

Price, G. R. 1972. Fisher's "fundamental theorem" made clear, Ann. Hum. Genet. 36, 129-140.

Price, G. R., and Smith, C. A. B. 1972. Fisher's Malthusian parameter and reproductive value, Ann. Hum. Genet. 36, 1-7.

Robertson, A. 1966. A mathematical model of the culling process in dairy cattle, Anim. Prod. 8, 95-108.

Turner, J. R. G. 1970. Changes in mean fitness under natural selection, in "Mathematical Topics in Population Genetics" (K. Kojima, Ed.), pp. 32-78, Springer-Verlag, Berlin. 